\title{
Hidden Caldeira-Leggett dissipation in a Bose-Fermi Kondo model
}

\author{
Mei-Rong $\mathrm{Li}^{1}$, Karyn Le Hur ${ }^{1}$, and Walter Hofstetter ${ }^{2}$ \\ ${ }^{1}$ Département de Physique and RQMP, Université de Sherbrooke, Sherbrooke, Québec, Canada J1K 2R1 \\ 2 Institut für Theoretische Physik A, RWTH Aachen, Templergraben 55, 52056 Aachen, Germany
}

\begin{abstract}
We show that the Bose-Fermi Kondo model (BFKM), which may find applicability both to certain dissipative mesoscopic qubit devices and to heavy fermion systems described by the Kondo lattice model, can be mapped exactly onto the Caldeira-Leggett model. This mapping requires an ohmic bosonic bath and an Ising-type coupling between the latter and the impurity spin. This allows us to conclude unambiguously that there is an emergent Kosterlitz-Thouless quantum phase transition in the BFKM with an ohmic bosonic bath. By applying a bosonic numerical renormalization group approach, we thoroughly probe physical quantities close to the quantum phase transition.
\end{abstract}

PACS numbers: 71.27.+a, 72.15.Qm, 75.20.Hr, 05.10.Cc

The Bose-Fermi Kondo model (BFKM) (or equivalently the spin-boson-fermion model), originally introduced by $\mathrm{Si}$ and coworkers $\frac{1}{2}$ and by Sengupta ${ }^{2}$ to describe peculiar quantum critical behaviors in heavyfermion Kondo lattice systems ${ }^{3}$, involves a single impurity spin being coupled both to a bosonic bath and to a fermionic bath. Resulting from the nontrivial competition between these distinct baths, a rich phase diagram is known to emerge from this model (See, e.g., Refs. 4 and 5). More generally, a great interest is currently devoted to the understanding of the Kondo entanglement breakdown mechanism due to the presence of extra (here, bosonic) quantum fluctuations resulting in striking quantum phase transitions ${ }^{6}$. In this Letter, we revisit the case where the impurity spin $S=1 / 2$ is coupled to an ohmic bosonic bath with a continuum spectrum - ohmic means that the bosonic correlation function in time $t$ decays as $1 / t^{2}$ - through an Ising coupling. The anisotropic Hamiltonian under consideration thus takes the form:

$$
\begin{aligned}
H= & h S_{z}+H_{s f}+H_{s b}, \\
H_{s f}= & \frac{J_{\perp}}{2}\left(\Psi_{\downarrow}^{\dagger}(0) \Psi_{\uparrow}(0) S_{+}+\text {h.c. }\right) \\
& +v_{f} \sum_{\sigma=\uparrow, \downarrow} \int_{-\infty}^{\infty} d x \Psi_{\sigma}^{\dagger}(x) i \partial_{x} \Psi_{\sigma}(x), \\
H_{s b}= & -\frac{v_{b} \partial_{x} \Phi(0)}{\sqrt{2 K_{b}}} S_{z}+\frac{v_{b}}{4 \pi} \int_{-\infty}^{\infty} d x\left[\partial_{x} \Phi(x)\right]^{2},
\end{aligned}
$$

where $h$ is a magnetic field, $\Psi_{\sigma}(x)$ and $\Phi$ represent the fermionic and bosonic fields; $v_{b}$ is the velocity of the bosons and $K_{b}^{-1} \neq 0$ the typical coupling between the bosons and the impurity spin; Recall that $K_{b}^{-1}=0$ means no coupling between the impurity spin and the bosonic environment. In Eq. (2), $v_{f}$ is the Fermi velocity. Without loss of generality, the one-dimensional character of the Hamiltonian $H_{s f}$ can be viewed as a result of the point-like character of the impurity and the rotational symmetry. Besides, we omit the Ising part of the Kondo coupling $J_{z}$ due to its minor effect (see the last page).

Our interest in the model (113) is also motivated by the fact that this model may be realized in mesoscopic dissipative setups involving qubits, as pointed out by one of us recently ${ }^{7}$. More precisely, the impurity spin can embody the two allowed charge states of a big metallic grain close to a given degeneracy point and $h$ being proportional to the gate voltage measures deviations from this degeneracy point ${ }^{8}$. The conduction electrons stand for the electrons both in the metallic grain $\left(\Psi_{\downarrow}\right)$ and in a nearby reservoir electrode $\left(\Psi_{\uparrow}\right)$, and the $J_{\perp}$ (Kondo) term denotes the tunneling process of an electron from lead to grain that flips (through the raising operator $S_{+}$) the charge state of the grain, and vice-versa. Note that here the spin index $\sigma=(\uparrow, \downarrow)$ is completely artificial and refers to the position of an electron in the structure (lead or grain). The original spin of the electrons are assumed to be polarized ("spinless electrons") due to the application of a strong magnetic field. The bosons represent the electromagnetic noise in the gate voltage stemming from the finite resistance $R$ in the gate lead, and the spinboson coupling reflects the effect of the voltage noise on the charge fluctuations of the grain. Here, $K_{b}=R_{K} / 2 R$ with $R_{K}=2 \pi \hbar / e^{2}$ the quantum of resistance $\underline{\underline{7}}$. Two of us have extended this model to one-dimensional reservoir leads being embodied by a Luttinger liquid behavior ${ }^{9}$ and also to two strongly capacitively-coupled large quantum dots (which can be reduced to a single charge qubit) ${ }^{10}$.

As a first step, the model of Eqs. (13) has been extensively studied $4,5,7$ by using a perturbative renormalization group (RG) approach. A quantum phase transition is discovered at the critical value of $K_{b^{7}}{ }^{7.11}$ :

$$
\left(K_{b}\right)_{c}^{-1}=J_{\perp} / \pi v_{f}=2 \Delta / \omega_{c},
$$

where $\omega_{c}$ is the high energy cutoff in the theory and $\Delta=$ $J_{\perp} \omega_{c} / 2 \pi v_{f}$. The RG flow equations suggest that the phase transition is of the Kosterlitz-Thouless (KT) type. On the other hand, a more rigorous approach is required in order to unambiguously prove the KT transition as well as to scrutinize the evolution of physical quantities in the vicinity of the quantum phase transition. In this Letter, we explore an exact mapping of the BFKM in Eqs. (13): We properly demonstrate that this model can be mapped onto the Caldeira-Leggett (CL) model12 with the effective dissipation strength

$$
\alpha=1+\left(4 K_{b}\right)^{-1}
$$


and therefore the two models belong to the same class of universality. It immediately follows that there is a KT quantum phase transition in the BFKM, separating a Kondo phase and an unscreened spin phase. Moreover, it irrefutably demonstrates the important Eq. (4). Even though the Bethe-Ansatz method ${ }^{13}$ can be exploited in the Kondo region, it breaks down in the vicinity of the quantum phase transition ${ }^{14}$ (which must be identified as the antiferromagnetic-ferromagnetic transition in the anisotropic Kondo model). We resort to a bosonic numerical RG (NRG) technique applying to the CL mode ${ }^{15}$. Of interest to us here is the spin magnetization at temperature $T=0,\left\langle S_{z}\right\rangle$, as well as the (local) spin susceptibility $\chi_{\text {loc }}(T)$ versus $T$. In the mesoscopic realizations, $\left\langle S_{z}\right\rangle=$ $\langle Q\rangle-1 / 2$, where $Q$ is the charge operator on the (large) dot, can be measured with very high precision ${ }^{7.9}$.

$C L$ mapping.-We first bosonize the fermions in Eq. (2) as: $\Psi_{\downarrow}^{\dagger}(x) \Psi_{\uparrow}(x)=\left[\omega_{c} /\left(2 \pi v_{f}\right)\right] \exp (i \sqrt{2} \varphi(x))$ and $\Psi_{\uparrow}^{\dagger}(x) \Psi_{\uparrow}(x)-\Psi_{\downarrow}^{\dagger}(x) \Psi_{\downarrow}(x)=\partial_{x} \varphi(x) /(\sqrt{2} \pi) ; \Delta$ and $\omega_{c}$ are defined in Eq. (4). By dropping the trivial contribution in the charge sector, the Hamiltonian $H_{s f}$ becomes

$H_{s f}=\frac{\Delta}{2}\left[e^{i \sqrt{2} \varphi(0)} S_{+}+h . c.\right]+\frac{v_{f}}{4 \pi} \int_{-\infty}^{\infty} d x\left[\partial_{x} \varphi(x)\right]^{2},(6)$

and the Hamiltonian (11) becomes that of an impurity spin coupled to two bosonic baths. Now we show that in the case of an ohmic bosonic bath in Eq. (3) one can envision to combine the two bosonic baths and eventually map the model onto a well-known CL model. The local action for the boson fields $\varphi(x)$ reads $S_{\varphi}^{l o c}=$ $\frac{T}{2 \pi} \sum_{\omega_{n}}\left|\omega_{n}\right| \varphi_{0}\left(\omega_{n}\right) \varphi_{0}\left(-\omega_{n}\right)$, where $\varphi_{0}=\varphi(x=0)$ and $\omega_{n}$ is the bosonic Matsubara frequency. Such a form implies that $\varphi(x)$ is of the ohmic type. If the bosonic bath $\Phi(x)$ in Eq. (3) is also ohmic and thus embodied by the local action $S_{\Phi}^{l o c}=\frac{T}{2 \pi} \sum_{\omega_{n}}\left|\omega_{n}\right| \Phi_{0}\left(\omega_{n}\right) \Phi_{0}\left(-\omega_{n}\right)$ with $\Phi_{0}=\Phi(x=0)$, we can then employ linear combinations of $\varphi_{0}$ and $\Phi_{0}$ to simplify the problem. (Note that starting from a non-ohmic bosonic environment ${ }^{16}$ instead, one would replace $\left|\omega_{n}\right|$ in $S_{\Phi}^{\text {loc }}$ by $\left|\omega_{n}\right|^{s}$ with $s \neq 1$; the two bosonic baths thus would have different dynamics which would hinder the straightforward combination.) By performing the unitary transformation $\mathcal{U}_{1}=$ $\exp \left\{-i \Phi_{0} S_{z} / \sqrt{2 K_{b}}\right\}$ to Eqs. (19), (3) and (6), we can absorb the spin-boson coupling term in Eq. (3) into the transverse Kondo term $\Delta$ in Eq. (6). It is then convenient to introduce the symmetric and antisymmetric bosonic combinations $\varphi_{s}=(2 \alpha)^{-1 / 2}\left[\sqrt{2} \varphi_{0}+\left(2 K_{b}\right)^{-1 / 2} \Phi_{0}\right]$ and $\varphi_{a}=(2 \alpha)^{-1 / 2}\left[\left(2 K_{b}\right)^{-1 / 2} \varphi_{0}-\sqrt{2} \Phi_{0}\right]$, where the crucial parameter $\alpha$ has been defined in Eq. (5). The impurity spin gets only coupled to the $\varphi_{s}$ mode and it is enough to write down the $\varphi_{s}$-action

$$
\begin{aligned}
S_{\varphi_{s}} & =\frac{T}{2 \pi} \sum_{\omega_{n}}\left|\omega_{n}\right| \varphi_{s}\left(\omega_{n}\right) \varphi_{s}\left(-\omega_{n}\right) \\
& -\frac{\Delta}{2}\left[e^{i \sqrt{2 \alpha} \varphi_{s}(0)} S_{+}+\text {h.c. }\right] .
\end{aligned}
$$

Conceptually, we can visualize $S_{\varphi_{s}}$ as the action linked to an Hamiltonian $H_{\varphi_{s}}$ with the effective velocity $v_{s}=\omega_{c} a$ ( $a$ is the short-distance cutoff which will be used below) and modeling a single bosonic bath with the dissipative parameter $\alpha$ coupled to the impurity spin. The link with the CL model of a two-level system with ohmic dissipation ${ }^{12}$ becomes clear when applying the unitary transformation $\mathcal{U}_{2}=\exp \left\{i \sqrt{2 \alpha} \varphi_{s}(0) S_{z}\right\}$ to turn the $\Delta$ term into a transverse magnetic field $\Delta S_{x}$, leading to

$$
\widetilde{H}=H_{S}-\sqrt{2 \alpha} v_{s} \partial_{x} \varphi_{s}(0) S_{z}+\frac{v_{s}}{4 \pi} \int_{-\infty}^{\infty} d x\left[\partial_{x} \varphi_{s}(x)\right]^{2},(8)
$$

where $H_{S}=h S_{z}+\Delta S_{x}$ and $\alpha$ is given in Eq. (5).

This mapping is crucial because it irrefutably reveals an originally hidden correspondence between the BFKM of Eqs. (1-3) and the CL model in which the breakdown of the Kondo physics (quantum coherence) due to dissipation is clearly established. For example, a KT type transition was predicted in Ref. 12 in the context of ohmic dissipation and this has been solidly confirmed through the NRG, either by refermionizing the bosonic degrees of freedom and then mapping the CL model onto the anisotropic Kondo model 17 , or directly by resorting to the bosonic NRG ${ }^{15}$. For $\Delta \rightarrow 0$ the KT transition is known to occur at $\frac{12}{2} \alpha=\alpha_{c}=1$ implying in the BFKM a critical $\left(K_{b}\right)_{c}^{-1} \rightarrow 0$ from Eq. (5): since $\Delta \rightarrow 0$, the Kondo energy scale $T_{K}$ characterizing the emergence of a bound state between the local moment and the conduction electrons vanishes and thus the local moment remains unscreened whatever the coupling between the dissipative mode $\partial_{x} \Phi(0)$ and the local spin.

To understand more deeply the connection between the CL model and the Kondo phenomenon, we can proceed from Eq. (8) along the lines of Ref. 18 and precisely recover the anisotropic Kondo model. The effective Kondo parameters here are given by $J_{\perp}=\pi v_{s} \Delta / \omega_{c}$ and $J_{z}=4 \pi v_{s}(1-\sqrt{\alpha})$. Thus, one can clearly distinguish two phases; a Kondo (delocalized) realm when $J_{z}>-\left|J_{\perp}\right|$ and a ferromagnetic Kondo phase which embodies an unscreened (localized) moment when $J_{z}<-\left|J_{\perp}\right|$. This enables us to rigorously predict a $\mathrm{KT}$ phase transition in the BFKM when $J_{z}=-\left|J_{\perp}\right|$ which implies a critical $\alpha_{c}=1+\left(K_{b}\right)_{c}^{-1} / 4=1+\Delta /\left(2 \omega_{c}\right)$ when assuming $0<\Delta \ll \omega_{c}$; this reinforces the intuition gained from the perturbative RG analysis of the BFKM ${ }^{7}$. Since BetheAnsatz calculations ${ }^{13}$ can only be applied to this model when $\alpha<\alpha_{c} \stackrel{14}{\underline{1}}$, we seek to apply the (bosonic) NRG on Eq. (8) to probe $\left\langle S_{z}\right\rangle$ versus $h$ and $\chi_{l o c}(T)$ very close to $\alpha_{c}$. Those quantities have not been studied in Ref. 15.

$N R G$ endeavors close to the transition.-The strength of the NRG lies in its nonperturbative nature and the ability to resolve arbitrarily small energies 19.20 .21 . This allows to provide important information in the phase transition region. For convenience, the Hamiltonian (8) can be rewritten $\mathrm{as}^{22}$

$$
\widetilde{H}=H_{S}+\omega_{c} \int_{0}^{1} d \epsilon\left[-\sqrt{2 \alpha \epsilon}\left(a_{\epsilon}+a_{\epsilon}^{\dagger}\right) S_{z}+\epsilon a_{\epsilon}^{\dagger} a_{\epsilon}\right],
$$



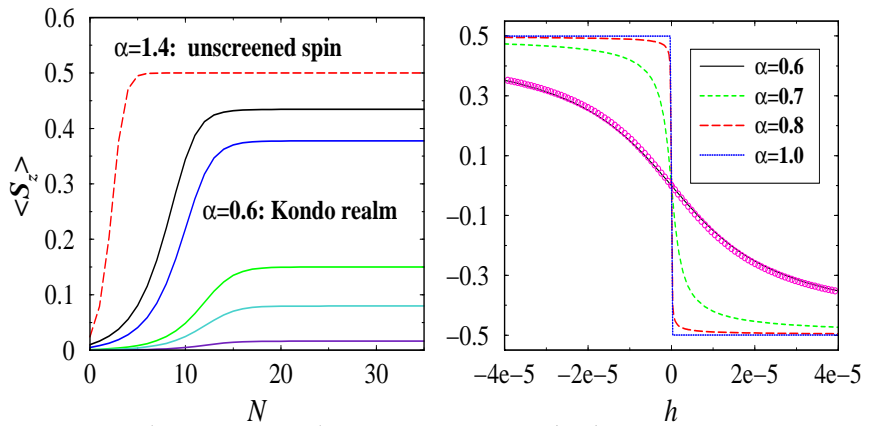

FIG. 1: (color online) Left: Flow of $\left\langle S_{z}\right\rangle$ with the iteration $N$. The dashed line is for $h=-0.0001$ (unscreened realm). All the solid lines correspond to $\alpha=0.6$ (Kondo regime), and from top to bottom, to $h=-10^{-4},-5 \times 10^{-5},-10^{-5},-5 \times$ $10^{-6},-10^{-6}$. Right: $\left\langle S_{z}\right\rangle$ as a function of $h$. The open circles sitting on top of the $\alpha=0.6$ curve are the Bethe-Ansatz results ${ }^{13,14}$. Parameters are $\Delta=0.01$ and $\omega_{c}=1$. The NRG parameters are $\Lambda=2, N_{s}=100, N_{b}=8$, and $N_{b 0}=500$.

Then, we introduce a logarithmic discretization of the energy interval $[0,1]$, i.e., a decreasing set of frequencies $\left(1, \Lambda^{-1}, \cdots, \Lambda^{-n}, \cdots\right)$ with $\Lambda>1$ and integer $n$. After transforming to a semi-infinite chain, $\widetilde{H}$ reads 15

$$
\begin{aligned}
H_{c}= & \lim _{N \rightarrow \infty} \Lambda^{-N} H_{N} \\
H_{N}= & \Lambda^{N}\left[H_{S}+\sqrt{\alpha} \omega_{c}\left(b_{0}+b_{0}^{\dagger}\right) S_{z}\right. \\
& \left.+\sum_{n=0}^{N} \epsilon_{n} b_{n}^{\dagger} b_{n}+\sum_{n=0}^{N-1} t_{n}\left(b_{n}^{\dagger} b_{n+1}+\text { h.c. }\right)\right] .
\end{aligned}
$$

In Eq. (10) the spin gets only coupled to the first (0th) site of the bosonic chain, and the remaining part of the chain is characterized by on-site energies $\epsilon_{n}$ and hopping parameters $t_{n}$. They satisfy a set of recursion relations which can be solved numerically. We refer the reader to Ref. 15 for the details of these recursion relations and their derivations as well as for the precise definition of the $b_{n}$ boson operators. It is important to note that both $\epsilon_{n}$ and $t_{n}$ decay exponentially as $\Lambda^{-n}$ as a result of the logarithmic discretization. This allows us to solve the model in Eq. (10) in an iterative way: first we diagonalize $H_{N}$ exactly, keep the $N_{s}$ lowest energy levels and then use the recursion relation between $H_{N+1}$ and $H_{N}$ derived from Eq. (11) to diagonalize $H_{N+1}$. Typically, the result converges at large $N \simeq 25$. In contrast to the fermionic case, each boson site allows for an infinite number of bosons and hence a truncation of the $\left(N_{b}+1\right)$ boson states is necessary ${ }^{23}$. Throughout this paper, we use $N_{s}=100$ and $N_{b}=8$ (except the 0th site for which $N_{b 0}=500$ ); those values are approximately those used in Ref. 15. We have also used another set of parameters $\left(N_{s}=150\right.$ and $\left.N_{b}=12\right)$ and found a good agreement. The quantities $\left\langle S_{z}\right\rangle(h)$ and $\chi_{\mathrm{loc}}(T)=d\left\langle S_{z}\right\rangle(T) /\left.d h\right|_{h \rightarrow 0}$ are also calculated iteratively.

$N R G$ Results.-In Fig. 囵(left) we show the flow of the ground state expectation value $\left\langle S_{z}\right\rangle$ as a function of the iteration variable $N$. At small $\Delta / \omega_{c}$, for $\alpha=1.4$ and for any $h<0$, after a few iterations we find that $\left\langle S_{z}\right\rangle$ flows

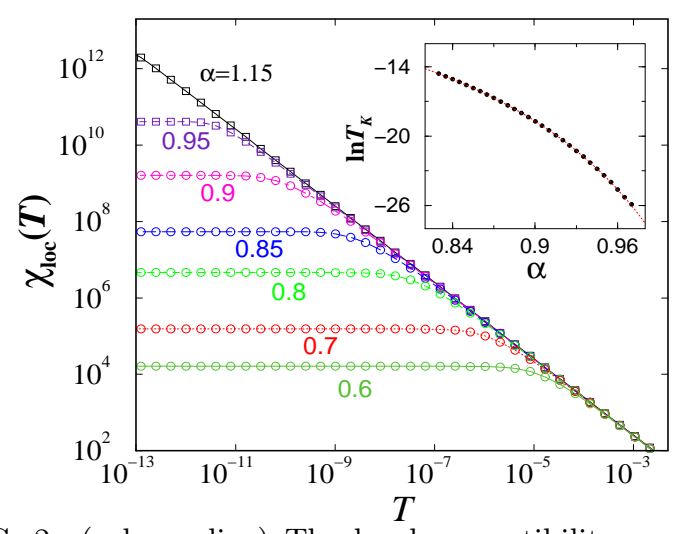

FIG. 2: (color online) The local susceptibility $\chi_{\text {loc }}(T)$ as a function of $T$. The open circles are the numerical data for $T=T_{N}=1.1 \Lambda^{-N}$ and lines are guides to the eyes. Inset: Logarithm of the Kondo energy scale $T_{K}$ versus $\alpha$ for $\alpha$ close to $\alpha_{c}$. The solid circles are the numerical data and the dashed line represents the fitting curve $\ln T_{K}=-4.67 /(1.15-\alpha)$. The NRG parameters, $\Delta$ and $\omega_{c}$, are the same as in Fig. 1]

rapidly to $1 / 2$, which is in agreement with the localized (dissipative) phase of the CL mode 12 as well as with the perturbative RG analysis of the BFKM ${ }^{7}$. This corresponds to an unscreened local spin or a particle which is localized in one of the two allowed levels. A strong dissipative Ising coupling with the bosonic bath definitely hinders the Kondo effect to develop. In contrast, for $\alpha=0.6$, we show that $\left\langle S_{z}\right\rangle$ flows to saturating values which deviate from $1 / 2$ and fall drastically with the decreasing $h$. Moreover, $\left\langle S_{z}\right\rangle$ yields a linear dependence versus $h$ around $h=0$ which indicates a fully screened moment: this is a clear signature of the Kondo effect. We have fitted the NRG curve for $\alpha=0.6$ (which is sufficiently far from $\alpha_{c}$ ) with Bethe-Ansatz results applying to the Kondo realm ${ }^{13.14}$. The dependence of $\left\langle S_{z}\right\rangle$ on $h$ at various $\alpha$ for $\Delta=0.01$ and $\omega_{c}=1$ is displayed in Fig. 1

We have carefully followed the progressive destruction of the delocalized Kondo regime when approaching the quantum phase transition at different applied magnetic fields. More specifically, we can infer the Kondo temperature $T_{K}$ from $\left\langle S_{z}\right\rangle$ versus $h$ based on the following scaling argument. The (Kondo) Fermi liquid type state emerging for $\alpha<\alpha_{c}$ is embodied by a constant (local) spin susceptibility $\chi_{\text {loc }}=\partial\left\langle S_{z}\right\rangle / \partial h$ which must be identified as ${ }^{7.13}$ $\chi_{\text {loc }} \approx 1 / T_{K}$. For $\alpha$ not too far from $\alpha_{c}$, we can then extract $T_{K}$ as the width of $h$ for $\left\langle S_{z}\right\rangle$ changing from -0.45 to 0.45 . This NRG procedure is complementary to that from the energy spectrum flow 15 . We check that the resulting $T_{K}$ obeys $\ln T_{K} \propto 1 /\left(\alpha_{c}-\alpha\right)$ close to $\alpha_{c}$ as clearly shown in the inset of Fig. 2 for $\Delta=0.01$ and $\omega_{c}=1^{24}$. The fact that $T_{K}$ decreases exponentially fast close to $\alpha_{c}$ is definitely reminiscent of the KT transition. The scaling in the inset of Fig. 2 also reveals that for the used NRG parameter $\Lambda=2, \alpha_{c}=1.15$. In fact, the NRG result is only exact in the limit of $\Lambda \rightarrow 1$. Hence, the precise $\alpha_{c}$ can be obtained by getting $\alpha_{c}$ for a few $\Lambda$ parameters and by extrapolating the result to $\Lambda \rightarrow 1$. For $\Delta / \omega_{c}=0.01$, 
we reach $\alpha_{c}(\Lambda \rightarrow 1)=0.98(\simeq 1)$. The effect of the dissipative coupling becomes weaker when increasing $\Delta$. For each $\Delta$, we have repeated the above-mentioned extrapolation procedure and precisely extracted $\alpha_{c}$ which basically corresponds to the critical bosonic coupling at which the Kondo scale strictly vanishes. The resulting $\alpha_{c}$ as a function of $\Delta$ obeys $\alpha_{c}(\Delta)=0.974+0.537 \Delta / \omega_{c}$ which clearly agrees with our expectation from the CL mapping. The formation of a Fermi liquid ground state in the Kondo realm is also clearly shown in Fig. 2 through the $\chi_{\text {loc }}(T)$ versus $T$ plot: For $\alpha<\alpha_{c}=1.15, \chi_{\text {loc }}(T)$ saturates to a constant for $T<T_{K}$ whereas for $T$ above $T_{K}$ the Curie's law $\chi_{\text {loc }} \propto 1 / T$ is well satisfied. At the transition point, we clearly observe that the $\chi_{\text {loc }}(T) \propto 1 / T$ behavior persists down to the lowest temperatures.

Finally, we would like to briefly comment on the effect of the Ising coupling term $J_{z}$ between the spin and the fermions, originally present in the heavy fermion systems but absent in the mesoscopic realizations under consideration, close to the phase transition point. For $\alpha \rightarrow 1$, $\varphi \simeq \varphi_{s}$ and thus the second term in the right hand side of Eq. (8) becomes $\sqrt{2}\left(\frac{1}{4 \pi} J_{z}-\frac{\sqrt{\alpha}}{2} v_{s}\right) \partial_{x} \varphi_{s}(0) S_{z}$. This immediately leads to a critical $\alpha_{c} \simeq 1+\mathcal{O}(\Delta)+\mathcal{O}\left(J_{z}\right)$, which is qualitatively consistent with Ref. 25 .
Conclusion.-We have demonstrated that the BFKM, with an ohmic bosonic bath and an Ising-type coupling between the latter and the impurity spin, and the CL model belong to the same class of universality. This unambiguously proves the existence of a KT phase transition at zero temperature in the former model when tuning the Ising dissipative coupling. We have chosen to resort to the bosonic NRG to investigate in detail physical quantities like the spin magnetization $\left\langle S_{z}\right\rangle$ versus the magnetic field $h$ close to the quantum phase transition where Bethe-Ansatz calculations break down. The latter clearly reflects two phases: the "localized" phase typical of an unscreened moment and the "delocalized" Kondo realm characterized by a Kondo energy scale which goes to zero exponentially fast close to the KT transition. Comparing with the recent fermionic NRG of the $\mathrm{BFKM}^{25}$, the bosonic NRG approach can be extended to the case of a sub-ohmic bath ${ }^{15,26}$ which might be relevant for the quest of quantum criticality in heavy-fermion systems ${ }^{27}$.

M.-R. Li thanks R. Bulla for discussions on the bosonic NRG. K.L.H. was supported by CIAR, FQRNT, and NSERC. K.L.H. thanks hospitality of SPHT Saclay and of KITP (Santa-Barbara) through the "Quantum phase transition" workshop, April 2005 (NSF PHY99-07949).
${ }^{1}$ Q. Si, S. Rabello, K. Ingersent, and L. Smith, Nature 413, 804 (2001) and Phys. Rev. B 68, 115103 (2003); J. L. Smith and Q. Si, Europhys. Lett. 45, 228 (1999).

2 A. M. Sengupta, Phys. Rev. B 61, 4041 (2000).

${ }^{3}$ H. von Löhneysen et al., Phys. Rev. Lett. 72, 3262 (1994); G. R. Stewart, Rev. Mod. Phys. 73, 797 (2001).

4 L. Zhu and Q. Si, Phys. Rev. B 66, 024426 (2002).

${ }^{5}$ G. Zaránd and E. Demler, Phys. Rev. B 66, 024427 (2002).

${ }^{6}$ P. Coleman, C. Pépin, Q. Si, and R. Ramazashvhili, J. Phys. Condens. Matter 13, R723 (2001); J. Custers et al., Nature, 424, 524-527 (2003); T. Senthil, S. Sachdev, and M. Vojta, Phys. Rev. Lett. 90, 216403 (2003).

7 K. Le Hur, Phys. Rev. Lett. 92, 196804 (2004).

${ }^{8}$ K. A. Matveev, Zh. Eksp. Teor. Fiz. 98, 1598 (1990) [Sov. Phys. JETP 72, 892, (1991)].

9 Karyn Le Hur and Mei-Rong Li, cond-mat/0410446

10 Mei-Rong Li and Karyn Le Hur, Phys. Rev. Lett. 93, 176802 (2004).

11 Following the perturbative RG of Ref. 7, we get the critical resistance $R_{c}=2 J_{\perp} N R_{K} / \nu=R_{K} /\left(2 K_{b}\right)$ where $N=1 /\left(2 \pi v_{f}\right)$ is the fermionic density of states and due to the definition of the boson operator $\partial_{x} \Phi(0)$ here $\nu=2$. The perturbative RG also predicts $T_{K}=\omega_{c} \exp \left[-4 /\left(\alpha_{c}-\alpha\right)\right]$ which resembles the fit of Fig. 2 (inset).

12 A. O. Caldeira and A. J. Leggett, Ann. Phys. 149, 374 (1983); A. J. Leggett et al., Rev. Mod. Phys. 59, 1 (1987).

13 A. M. Tsvelik and P. G. Wiegmann, Adv. Phys. 32, 453 (1983); N. Andrei, K. Furuya, and J. H. Lowenstein, Rev. Mod. Phys. 55, 331 (1983).

14 P. Cedraschi and M. Büttiker, Ann. Phys. 289, 1 (2001).
15 R. Bulla, N.-H. Tong, and M. Vojta, Phys. Rev. Lett. 91, 170601 (2003); cond-mat/0407559

16 D. Grempel and Q. Si Phys. Rev. Lett. 91, 026401 (2003).

17 T. Costi, Phys. Rev. Lett. 80, 1038 (1998).

18 A. Furusaki and K. A. Matveev, Phys. Rev. Lett. 88, 226404 (2002).

19 K. G. Wilson, Rev. Mod. Phys. 47, 773 (1975).

${ }^{20}$ H. R. Krishna-murthy, J. W. Wilkins, and K. G. Wilson, Phys. Rev. B 21, 1003 (1980); ibid. 21, 1044 (1980).

21 W. Hofstetter, Phys. Rev. Lett. 85, 1508 (2000).

22 From bosonization, $\varphi_{s}(x)=-\sqrt{\frac{2 \pi}{L}} \sum_{q>0} \frac{1}{i \sqrt{q}}\left(a_{q} e^{i q x}-\right.$ h.c.) $e^{-a q / 2}$, where $L$ is the length of the system and $a$ is a typical short-distance cutoff; note that $\left[\partial_{x} \varphi_{s}(x), \varphi_{s}(y)\right]=i 2 \pi \delta(x-y)$. This leads to $\widetilde{H}=H_{S}+$ $2 \sqrt{\alpha} v_{s} \sqrt{\frac{\pi}{L}} \sum_{q>0} \sqrt{q}\left(a_{q}+a_{q}^{\dagger}\right) e^{-a q / 2} S_{z}+v_{s} \sum_{q>0} q a_{q}^{\dagger} a_{q}$. Rescaling $q=\epsilon / a$ and equating the high-energy cutoff $\omega_{c}=v_{s} / a$, we reach Eq. (9) with $\left[a_{\epsilon}, a_{\epsilon^{\prime}}^{\dagger}\right]=\delta\left(\epsilon-\epsilon^{\prime}\right)$.

23 R. V. Pai et al., Phys. Rev. Lett. 76, 2937 (1996); L. G. Caron and S. Moukouri, ibid. 76, 4050 (1996).

24 The mapping requires $\alpha \rightarrow 1$. So to check the result in Eq. (4), we must examine $T_{K}$ for $\alpha$ close to $\alpha_{c}$. For $\alpha$ far away from $\alpha_{c}$ there is a similar scaling $\ln T_{K} \propto 1 /\left(\alpha_{c}-\right.$ $\alpha)^{15,17}$, but the prefactor $\ln \left(\Delta / \omega_{c}\right)$ is not equal to $-4^{11}$.

${ }^{25}$ L. Borda, G. Zaránd, and P. Simon, cond-mat/0412330

26 M.-R. Li, Ph. Doucet-Beaupré, K. Le Hur, and W. Hofstetter, in progress.

27 M. T. Glossop and K. Ingersent, cond-mat/0501601 